\title{
7 Sprach- und gestenwissenschaftlicher Bezugspunkt: Metapher als dynamischer, situativ verorteter und verkörperter Denkprozess
}

Kognitive Metapherntheorien, die an einer Gebrauchsperspektive orientiert sind, gibt es nicht erst, seit sich in Resonanz auf die Konzeptuelle Metapherntheorie, welche auf der Ebene von Sprachgemeinschaften zu verorten ist, die Kritik formiert hat, dass die konkreten Äußerungssituationen und -kontexte dort außer Acht gelassen sind. So adressiert etwa Max Blacks seit den 1950ern entwickelte Interaktionstheorie ${ }^{1}$ metaphorische Aussagen; das meint Aussagen, bei denen ein Fokus und ein Rahmen zusammenwirken. ${ }^{2}$ Solche Aussagen basieren Black zufolge weniger auf objektiv gegebenen Ähnlichkeiten - vielmehr kreieren sie Ähnlichkeiten. ${ }^{3}$ Metaphorische Aussagen werden als „,kognitive Instrumente“ charakterisiert, „die unerläßlich sind zur Wahrnehmung von Verbindungen, welche, einmal erkannt, dann wirklich vorhanden sind“ - in der „Herstellung der Metapher“ wird auf diese Weise ein wirklichkeitskonstituierendes Moment gesehen. ${ }^{4}$ Und eine solche Auffassung von der Metapher knüpft sich wiederum an die Überzeugung, ,daß die ,Welt‘ notwendig eine Welt in einer bestimmten Beschreibung ist - oder eine Welt, die aus einer bestimmten Perspektive gesehen wird“. 5 Metaphern wird das Vermögen zugesprochen, solche Perspektiven, ja: ,Welt‘ erzeugen zu können.

Der vorliegende Teil II, der ein film- und medienwissenschaftliches Verständnis von ,Metapher' im Kontext audiovisueller Bilder aus einer Gebrauchsperspektive entwickelt, nimmt Anschluss an diese sprachphilosophischen Überlegungen Blacks zu metaphorischen Aussagen - ist doch auch das filmische Bild nicht einfach als Repräsentation einer gegebenen Welt, sondern als spezifische Konstitutionsweise von Welt zu erachten. ${ }^{6}$ Wie soeben im vorangegange-

1 Black: Metaphor; Black: Mehr über die Metapher.

2 Black baut wiederum auf Richards rhetorisch orientierten Überlegungen aus den 1930er Jahren auf (Ivor Armstrong Richards: Metaphor [1936]. In: The Philosophy of Rhetoric. New York: Oxford University Press 1965, S. 89-114).

3 Vgl. Black: Metaphor, S. $284 \mathrm{f}$.

4 Black: Mehr über die Metapher, S. 409.

5 Ebd.

6 Vgl. etwa Gilles Deleuze: Das Bewegungs-Bild. Kino 1 [1983]. Frankfurt a. M.: Suhrkamp 1989; Deleuze: Das Zeit-Bild. Und signifikanter Weise ist es ein medientheoretischer Gedanke, mit dem 
nen Kapitel ,Perspektivverschiebungen“ skizziert, wird darüber hinaus der Annahme gefolgt, dass ein Gespräch und das Schauen eines Films gewisse Ähnlichkeiten zeigen, insofern beide als sich in der Zeit entfaltende, multimodale Diskursereignisse $\mathrm{zu}$ betrachten sind. Dies motiviert das Vorgehen, aus dem Bereich der Sprach- und Gesprächsforschung weitere grundlegende Annahmen zur Metapher aus einer Gebrauchsperspektive, wie sie sich maßgeblich seit Ende der 1990er Jahre entwickelt haben (und prägend für eine solche Perspektive in der linguistischen Metaphernforschung geworden sind), ausführlicher vorzustellen und einzuholen: Mit Lynne Cameron, deren Forschung sich auf sprachliche Äußerungen konzentriert, wird in den Blick genommen, dass und in welcher Weise ,Metapher' eine dynamische diskursimmanente und diskursgestaltende Aktivität ist - und eine Metaphernanalyse daher auch eine Möglichkeit bietet, sich Diskursereignisse zu erschließen. Mit Cornelia Müller, die das sich in der Zeit entfaltende Zusammenspiel von Sprache und Geste betrachtet, wird vor allem auf anschauliches Denken und geteilte Wahrnehmungen abgehoben. Wie solch linguistische Positionen ,Metapher' in der Alltagskommunikation als einen dynamischen, situativen und verkörperten Denk- und Verstehensprozess entwerfen, ${ }^{7}$ soll daher als metapherntheoretischer Bezugspunkt und auch erste methodische Orientierung für das zu entwickelnde Verständnis filmischen Metaphorisierens fungieren.

\subsection{Metapher als diskursimmanente Aktivität (Cameron)}

Die Linguistin Lynne Cameron, die sich für Sprachgebrauch und Verstehensprozesse in jeweils spezifischen dialogischen Kontexten interessiert, hat sich insbesondere mit Unterrichtsgesprächen ${ }^{8}$ und Gesprächen von Menschen in PostKonflikt-Gesellschaften ${ }^{9}$ beschäftigt. Beide Male zeigt sich die Metapher als ein dialogischer Verständigungsweg: sei es in Prozessen des Lernens oder in Prozessen der Versöhnung und Bildung von Empathie. Camerons Untersuchungen

\footnotetext{
Black sich seiner Idee einer kreativen, wirklichkeitskonstituierenden Metapher annähert: „,Hat es das Zeitlupenbild eines galoppierenden Pferdes vor der Erfindung der Kinematographie gegeben?' Hier wird die ,Sicht‘ notwendigerweise durch ein vom Menschen geschaffenes Instrument vermittelt [...]. Und doch wird das, was im Zeitlupenfilm zu sehen ist, sobald es erst einmal wahrgenommen wurde, zu einem Bestandteil der Welt.“ (Black: Mehr über die Metapher, S. 408.) 7 Siehe weiterführend hierzu auch Kappelhoff: Kognition und Reflexion sowie Müller/Kappelhoff: Cinematic Metaphor.

8 Lynne Cameron: Metaphor in Educational Discourse. London: Continuum 2003.

9 Cameron: Metaphor and Reconciliation.
} 
konzentrieren sich dabei auf die Verbalsprache, denn sie erachtet die Metapher nicht als etwas rein Kognitives, sondern als etwas, das zugleich grundlegend sprachlich ist. ${ }^{10}$ Doch selbst wenn hier ausschließlich sprachliche Äußerungen betrachtet werden, die ja beim audiovisuellen Bild nur eine (und nicht unbedingt notwendige) Artikulationsdimension betreffen: Camerons im sogenannten ,discourse dynamics'-Ansatz ${ }^{11}$ verankertes Metaphernverständnis ist für den vorliegenden Kontext relevant, weil ,Metapher‘ als ein in einem Diskursereignis emergierendes Phänomen und als eine Aktivität aufgefasst wird, weil es als etwas dezidiert Dynamisches, Prozessuales in den Blick genommen wird. So hat Cameron ein äußerst dynamisches Metaphernverständnis entwickelt, für das die Gebrauchsperspektive die maßgebliche ist:

Metaphor, [...] from the discourse dynamics perspective, becomes processual, emergent, and open to change. Rather than seeing metaphor as a tool or some other kind of object that is put to use, a processual view attends to metaphor activity. Metaphor is not part of a system that is put to use; from a dynamic perspective there is only use. ${ }^{12}$

Und noch die Rede vom ,Metapherngebrauch“ wird kritisch hinterfragt, suggeriere diese doch letztlich selbst, dass die Metapher ein zu gebrauchendes Objekt sei. Es liegt daher allein an der Tendenz der englischen Sprache, nominale Ausdrücke

10 Vgl. Lynne Cameron: Metaphor in Physical-and-Speech Action Expression. In: Researching and Applying Metaphor in the Real World. Hrsg. v. Graham Low, Zazie Todd, Alice Deignan und Lynne Cameron. Amsterdam: John Benjamins 2010, S. 333-355, hier S. 334.

11 Die Diskursform, Gespräch“ wird mit Bezug auf die Dynamic System Theory dabei als ein in der soziokulturellen Welt verankertes Ereignis betrachtet, wodurch ein Diskurs immer schon gewisse Ausgangskonditionen hat und zugleich aber auch auf die soziokulturelle Ebene Einfluss nimmt. Für eine ausführlichere Erläuterung der Idee, Gespräche in der Perspektive komplexer dynamischer Systeme zu fassen, vgl. Lynne Cameron: The Discourse Dynamics Framework to Metaphor. In: Metaphor Analysis. Research Practice in Applied Linguistics, Social Sciences and the Humanities. Hrsg. v. Lynne Cameron und Robert Maslen. London: Equinox 2010, S. 77-94, hier S. 82-93. Die Idee dynamischer Systeme ist den Naturwissenschaften entnommen - ein Beispiel für solch ein dynamisches System in der Umwelt ist etwa die Wolke, die aus der Interaktion von Wind, Feuchtigkeit und Temperatur emergiert. Für eine Übersicht zum Discourse DynamicsAnsatz vgl. auch Cameron: Metaphor in Physical-and-Speech Action Expression, S. 333-336. Vgl. darüber hinaus Diane Larsen-Freeman/Lynne Cameron: Complex Systems and Applied Linguistics. Oxford: Oxford University Press 2008; Gibbs/Cameron: The Social Cognitive Dynamics of Metaphor Performance.

12 Lynne Cameron/Robert Maslen/Zazie Todd/John Maule/Peter Stratton/Neil Stanley: The Discourse Dynamics Approach to Metaphor and Metaphor-Led Discourse Analysis. In: Metaphor and Symbol 24.2 (2009), S. 63-89, hier S. 67, Herv. CS. Vor allem Gibbs entwickelt ein Verständnis konzeptueller Metaphern, die diese an dynamische Gebrauchstheorien der Metapher annähert. Vgl. Gibbs: Metaphor Wars. 
den verbalen vorzuziehen, dass Cameron das ,Metaphorisieren“ als sprachliche Alternative zu ungelenk erscheint. Dennoch ist eben damit die Aktivität, die hier mit ,Metapher‘ adressiert wird, terminologisch präziser zu greifen: „We might describe a dynamic perspective more accurately with verbs such as ,metaphorising [...]. " ${ }^{13}$ Welche Aspekte eines Metaphorisierens nun mit Cameron in den Blick kommen, sei im Folgenden sondiert.

Cameron arbeitet mit Transkriptionen von Gesprächen. Solche Transkriptionen sind für sie eine Spur eines „talking-and-thinking“, also die Spur einer sich tatsächlich ereignet habenden diskursiven Aktivität, bei der Sprechen und Denken miteinander zusammenhängen. Das Diskursereignis selbst bestimmt sie als „a bounded and coherent human activity involving the use of language“ - beispielsweise ein zur Versöhnung anberaumtes Gespräch, eine Schulstunde, eine Radiosendung, ein Treffen, ein Arzt-Patienten-Gespräch, oder ein Gespräch zwischen Freunden/Kollegen beim Kaffee. ${ }^{14}$ Ein solches Diskursereignis wird als ein komplexes, dynamisches, selbst noch erst entstehendes System der im Gespräch interagierenden Personen betrachtet: „The dynamic system of discourse develops, adapts and flows as speakers' contributions build on each other, and as people develop their own or others' ideas. ${ }^{15}$ Und dies impliziert damit auch die Abkehr von einer statischen Idee von Bedeutung und Verstehen: „What if understanding through talk is achieved, not by choosing words to contain meanings and then placing meanings on the table for one's interlocutor to pick up, but rather is the result of the dynamics of the system?" 16

Sprachlich artikulierte Metaphern zu analysieren, hat sich dabei für Cameron als ein zentraler diskursanalytischer Zugang erwiesen:

Metaphor is everywhere once we look for it, or nearly everywhere; sometimes especially powerful and meaningful, but more often just mundane and ordinary. [...] Plotting people's metaphors as they talk is rather like following the breadcrumb trail that led Hansel and Gretel out of the forest. Metaphors offer a path through the confusion of conversation, with its stops and starts, its deviations and back tracks. ${ }^{17}$

13 Cameron/Maslen/Todd/Maule/Stratton/Stanley: Discourse Dynamics Approach to Metaphor, S. 67, Herv. CS. Vgl. auch Lynne Cameron: From Metaphor to Metaphorizing. How Cinematic Metaphor Opens Up Metaphor Studies. In: Cinematic Metaphor in Perspective. Reflections on a Transdisciplinary Framework. Hrsg. v. Sarah Greifenstein, Dorothea Horst, Thomas Scherer, Christina Schmitt, Hermann Kappelhoff und Cornelia Müller. Berlin/Boston: Walter de Gruyter 2018, S. 17-35.

14 Cameron: The Discourse Dynamics Framework to Metaphor, S. 93.

15 Ebd., S. 82.

16 Larsen-Freeman/Cameron: Complex Systems and Applied Linguistics, S. $10 \mathrm{f}$.

17 Cameron: Metaphor and Reconciliation, S. 4. 
Als ein wissenschaftlicher Zugang zu (oder, um im Märchenbild $\mathrm{zu}$ bleiben: Ausweg aus) Diskursen und ihrer Komplexität kann die Metaphernanalyse jedoch nur fungieren, wenn die Metapher selbst noch - als Diskursphänomen eben nicht als etwas Statisches, sondern als ein sich immer wieder veränderndes, dynamisches Phänomen aufgefasst wird. ,Metapher‘ in Gesprächen meint dann nicht nur das Zusammenbringen zweier verschiedener Ideen, aufgrund derer semantischer Interaktion etwas über diese beiden für sich genommenen Ideen Hinausgehendes entsteht: ${ }^{18}$ Es meint darüber hinaus etwas, das den Gesprächsverlauf formt und selbst von diesem geformt wird, es meint ,a shifting, dynamic phenomenon that spreads, connects, and disconnects with other thoughts and other speakers, starts and restarts, flows through talk developing, extending, changing“. ${ }^{19}$ Eben diese ,amorphous and shifting nature of metaphor in use“ scheint der Normalfall von Metaphern in Gesprächen zu sein und verlangt, auch theoretisch zentrale Berücksichtigung zu finden. ${ }^{20}$

Anders als dies etwa bei Forceville der Fall ist, ${ }^{21}$ verortet Cameron vor diesem Hintergrund die metaphorische Kreativität denn auch auf der Gebrauchsebene, d. h. mit Blick auf die konkrete diskursive Ausgestaltung und Erscheinungsweise (weshalb auch die in den meisten Gesprächen beobachtbare Verwendung mehr oder weniger konventionalisierter metaphorischen Redensarten kein Ausschluss von Kreativität ist):

The creativity of metaphor in talk appears less in the novelty of connected domains and more in the use of metaphor to shape a discourse event and in the adaption of metaphor in the flow of talk. Metaphor in talk is not evenly spread but gathers in clusters and occasionally, and then significantly, is altogether absent. ${ }^{22}$

Aus discourse dynamics-Perspektive lassen sich Metaphern im Kontext diskursiver Gesprächsereignisse maßgeblich als Emergenzprozesse beschreiben, als etwas, das sich entfaltet, sich verändert und im Verlauf des Ereignisses Muster ausbildet. Diese Prozesse, diese Bewegungen im Metaphorisieren fasst Cameron zum einen über das sogenannte metaphor shifting. ${ }^{23}$ Damit wird beschreibbar, wie

18 So das basale Metaphernverständnis Camerons (ebd., S. 3) mit Bezug auf die Interaktionstheorie der Metapher nach Richards.

19 Lynne Cameron: Metaphor and Talk. In: The Cambridge Handbook of Metaphor and Thought. Hrsg. v. Raymond W. Gibbs, Jr. Cambridge: Cambridge University Press 2008, S. 197-211, hier S. 197.

20 Cameron: Metaphor in Physical-and-Speech Action Expression, S. 336.

21 Vgl. hierzu Kapitel 3 der vorliegenden Arbeit.

22 Cameron: Metaphor and Talk, S. 197.

$23 \mathrm{Zu}$ Dynamiken von Metaphernemergenzen vgl. Cameron: The Discourse Dynamics Framework to Metaphor, S. 87-93. 
im Kontext eines Diskursereignisses einzelne metaphorisch gebrauchte Wörter und Phrasen - sogenannte Metaphern-Vehikel - zusammenhängen. (In dezidierter Abgrenzung zur KMT, und in Anlehnung an Richards, Black u. a. spricht Cameron weniger von source und target, sondern vielmehr von vehicle und topic: Metaphern-Vehikel weisen auf die Gegenwart einer Metapher hin, sind jedoch nur ein Teil der Metapher: Der andere Teil ist das metaphorische topic.) Aufgrund von diversen Studien sind vor allem drei Shifting-Typen ausgemacht worden: ${ }^{24}$

- Verlagerung eines metaphorischen Vehikels von einem metaphorischen Thema (topic) zu einem anderen

- Entwicklung eines metaphorischen Vehikels durch

- Wiederholung

- Re-Formulierung, etwa durch Synonymverwendung

- Explizierung im Sinne von Ausdehnung, Elaborierung, Exemplifizierung

- Kontrastierung

- Ent-Metaphorisieren eines zuvor metaphorischen Vehikels durch WörtlichNehmen

Gerade mit der Entwicklung eines metaphorischen Vehikels kann Cameron zwei Phänomene in den Blick nehmen: die ausgedehnte Metapher ${ }^{25}$ und die von Cameron so genannte systematische Metapher:

[...] the outcome of intensive elaboration or explication of a particular vehicle over a short period of time will appear in the trace of discourse as an ,extended metaphor. Over longer periods of time, such as an entire discourse event, successive episodes and instances of vehicle developement around closely connected topics produce what we call ,systematic metaphor'. [...] A systematic metaphor is not a single metaphor but an emergent grouping of closely connected metaphors. ${ }^{26}$

Beispielhaft für die Entwicklung eines metaphorischen Vehikels, die auf das ganze Diskursereignis ausgreift, wodurch dieses besondere metaphorische Diskursmuster der ,systematic metaphor` emergiert, sei hier auf die Untersuchung eines Fokusgruppengesprächs zu Terrorismusrisiko verwiesen:

24 Vgl. für eine ausführlichere Übersicht der Typen des Metaphern-Shifting im dialogischen Sprachgebrauch Abb. 49 (im Anhang) sowie für eine Herleitung und Diskussion dieser Typen Lynne Cameron: Metaphor Shifting in the Dynamics of Talk. In: Confronting Metaphor in Use. An Applied Linguistic Approach. Hrsg. v. Mara Sophia Zanotto, Lynne Cameron und Marilda C. Cavalcanti. Amsterdam: John Benjamins 2008, S. 45-62.

25 Vgl. hierzu z. B. auch Paul Werth: Extended Metaphor - a Text-World Account. In: Language and Literature 3.2 (1994), S. 79-103.

26 Cameron: The Discourse Dynamics Framework to Metaphor, S. $90 \mathrm{f}$. 


\begin{abstract}
The risk was characterized as a poker game and a game of bluff involving lottery odds for a public who were pawns in a game. One participant observed that if the worst happened, my number's up. Thinking of it from the perspective of the authorities as players in such games, another said, they're actually dicing with your life. What all these metaphors have in common is not just that they relate to games, but that the games have outcomes which affect the public in arbitrary ways; in other words, they are games of chance. These individual metaphors therefore contribute to a systematic metaphor we can formulate as BEING AFFECTED BY TERRORISM IS PARTICIPATING IN A GAME OF CHANCE. ${ }^{27}$
\end{abstract}

Eine solche systematische Metapher ist Cameron zufolge ein kontext-gebundenes Phänomen, das immer in Beziehung zu einem spezifischen Diskursereignis zu begreifen ist: „Systematic metaphors are emergent phenomena that arise on the level of the discourse event out of decisions made in the minute-by-minute dynamics of talking-and-thinking, and are identified inductively.“28 Das unterstreicht noch einmal die Differenz systematischer Metaphern im Vergleich zu generalisierten konzeptuellen Metaphern, sind letztere doch „abstracted across multiple discourse events and speakers“. ${ }^{29}$ Die Fluchtlinie einer systematischen Metapher hingegen sind spezifische, situativ verortete Denkprozesse, gebunden an das jeweilige Diskursereignis. Die Idee systematischer Metaphern ist es, aufzuzeigen, wie Teilnehmer eines Gesprächs (als einem emergierenden dynamischen System) gemeinsam in spezifischer Weise ein spezifisches Thema darstellen. Um mit einer systematischen Metapher analytisch reflektieren zu können, wie sich im Verlauf eines Gesprächs ein Bedeutungsganzes aus zunächst einzeln identifizierten sprachlichen Metaphern fügt, ist es wichtig, mit ihrer Formulierung möglichst nah am gegebenen Sprachmaterial zu bleiben und zugleich die übergreifende Idee zu erfassen. ${ }^{30}$

Zwar verbindet sich sowohl mit dem Begriff der konzeptuellen Metapher als auch mit jenem der systematischen Metapher der Anspruch, metaphorische Muster im Denken zu reflektieren. Und beide Begriffe gehen davon aus, dass solche metaphorischen Muster (die KMT spricht in der Regel von Strukturen) Relevanz

27 Lynne Cameron/Graham Low/Robert Maslen: Finding Systematicity in Metaphor Use. In: Metaphor Analysis. Research Practice in Applied Linguistics, Social Sciences and the Humanities. Hrsg. v. Lynne Cameron und Robert Maslen. London: Equinox 2010, S. 116-146, hier S. 117. Mit der Schreibweise systematischer Metaphern in kleinen, kursivierten Kapitälchen wird sich von konzeptuellen Metaphern abgegrenzt.

28 Cameron: Metaphor Shifting in the Dynamics of Talk, S. 57. Vgl. auch Cameron/Low/Maslen: Finding Systematicity in Metaphor Use.

29 Cameron: Metaphor Shifting in the Dynamics of Talk, S. 57.

30 Vgl. Cameron/Low/Maslen: Finding Systematicity in Metaphor Use, S. 117. Vgl. auch Cameron: Metaphor in Physical-and-Speech Action Expression, S. 333f. 
für das Verstehen, Sprechen, Schreiben usw. haben. ${ }^{31}$ Trotz dieser Gemeinsamkeiten unterscheiden sich die beiden Metaphernauffassungen in ihrer theoretischen Anlage jedoch grundlegend:

In the discourse dynamics framework, people's language and cognitive resources are seen as prior to their participation in discourse events, and these resources may include conventionalized ways of thinking-and-talking, but no priority is given to thought over language or to the general over the specific. ${ }^{32}$

Wenn Cameron über das Gruppieren von Metaphern ${ }^{33}$ metaphorische Muster zu identifizieren sucht, dann werden diese jeweils auf ein Diskursereignis bezogenen Muster (,patterns“) daher gerade nicht als fixierte, präexistente Denkstrukturen adressiert, die spezifische (sprachliche) Äußerungen instanziieren. Vielmehr sind die metaphorischen Diskursmuster etwas im Prozess der Artikulation Entstehendes, etwas Fluides und auch teils sehr Subtiles, Angespieltes. Sie sind, so könnte man sagen, konstruktive Oberflächenphänomene: Die Muster sind unablösbar von ihrer Erscheinungsweise.

Neben dem shifting-Befund - dass also ein Vehikel-Ausdruck sich dialogisch verlagert, verschiebt, bewegt und seine Form im Gesprächsverlauf verändert - ist noch ein weiterer zentraler Befund von Studien spontaner Gespräche zu nennen, der das Emergente und Dynamische des Metaphorisierens anzeigt: Das metaphorische Thema (topic) bleibt häufig implizit, sodass sich nicht für jedes vehicle ein dezidiertes Thema bestimmen lässt: „the vehicle term usually comes ,on its own“, rather than linked to an explicit topic term (in contrast to the nominal form of literary metaphors such as Juliet is the sun, or conceptual metaphors in the form of a is b)“ ${ }^{34}$ Das metaphorische topic ist damit als ein häufig vage bleibender Prozess im Gesprächsfluss zu beschreiben; es ist etwas, das „,in der Kommunikation selbst überhaupt erst erzeugt“" wird. ${ }^{35}$

31 Vgl. Cameron: The Discourse Dynamics Framework to Metaphor, S. 91.

32 Ebd., Herv. CS.

33 „The first step in grouping metaphors across time is to code vehicles by their semantic content, their more basic or physical meaning. After that, within each collected grouping of vehicles, a further sub-grouping is carried out, this time by key discourse topic. The outcome of this process of grouping and sub-grouping is the set of metaphor trajectories or systematic metaphors.“ (Cameron: Metaphor and Reconciliation, S. 45.) Vgl. auch Abb. 50 (im Anhang) für eine kurze Beispielanalyse.

34 Cameron: Metaphor in Physical-and-Speech Action Expression, S. 333 f.

35 So Schröder in ihrer Darstellung von Camerons Ansatz (Schröder: Kommunikationstheoretische Fragestellungen in der kognitiven Metaphernforschung, S. 102). Cameron zufolge lassen sich zumeist sogenannte „key discourse topics“ beschreiben, deren analytische Benennung teils tentativ bleiben muss. 
Und schließlich sei noch eine sehr grundsätzliche These Camerons aufgegriffen: Eine Metapherntheorie, die monologisch auf die Welt blickt und dem Individualistischen verhaftet ist, wird als problematisch erachtet. Metaphorische Phänomene, wie sie sich in „real-world-contexts“ zeigen, ließen sich damit nicht verstehen, nicht erklären - insofern die Welt doch grundsätzlich sozial, dialogisch ist und auf dynamischen Austauschprozessen basiert. ${ }^{36}$ Weite Teile der Metapherntheorie jedoch, so Camerons Befund, haben eine monologische, individualistische Tendenz: So ist die klassische Metapherntheorie ${ }^{37}$ in der Literaturtheorie verwurzelt und hat sich insofern mit Texten beschäftigt, nicht mit Interaktionen. Und auch die Konzeptuelle Metapherntheorie wird aus zweierlei Gründen als individualistisch angelegt charakterisiert: Zum einen, weil sich die KMT aus dem individualistischen generativen Sprachmodell entwickelt hat - als Abgrenzungsbewegung zwar (insofern das Moment der Erfahrung stark gemacht wird), jedoch ohne dabei das Moment des Individualistischen zu kritisieren. Zum anderen wird angeführt, welch starke Rolle für die KMT das weitere Feld der ebenfalls wesentlich von einem monologischen Modell geprägten Kognitionspsychologie spielt. $^{38}$

\subsection{Aktivierung von Metaphorizität in verbal-gestischer Interaktion (Müller)}

Die Gesten- und Sprachwissenschaftlerin Cornelia Müller hat in ihrer breit angelegten, grundsätzlichen Auseinandersetzung mit dem Gebrauch von Sprache ebenfalls ein dynamisches Verständnis von Bedeutung und Metapher entwickelt. Dabei wird eine Ebene in die Idee von Metapher als einem dynamischen Sprachgebrauchsphänomen einbezogen, die für die vorliegende Studie zentral ist: Wo Cameron sich rein auf Sprachäußerungen im Diskursereignis konzentriert, erweitern Müllers Forschungen den Blick zu multimodaler Interaktion, insofern solche Äußerungen in der Face-to-Face-Kommunikation als Teil eines verbal-gestischen, d.h. multimodalen Artikulationskontexts betrachtet werden. Denn „viele [...] sprachliche Äußerungen sind in Alltagsgesprächen gestisch begleitet“, Sprechen

36 Vgl. Cameron: Metaphor in Physical-and-Speech Action Expression, S. 334.

37 Hierzu gehören auch Max Black: Models and Metaphors. New York: Cornell University Press 1962; Ivor Armstrong Richards: Die Metapher [1936]. In: Theorie der Metapher. Hrsg. v. Anselm Haverkamp. Darmstadt: Wissenschaftliche Buchgesellschaft 1983, S. 31-52.

38 Vgl. Cameron: Metaphor in Physical-and-Speech Action Expression, S. 334. Vgl. hierzu auch die Ausführungen zur KMT in Kapitel 2 der vorliegenden Arbeit. 
ist das „primäre und ,natürliche“ Umfeld“ von Gesten: ${ }^{39}$ „Gesten, gefasst als kommunikative Bewegungen der Hände, treten primär gemeinsam und in Einklang mit der Lautsprache auf. Sie sind auf dieses Zusammenspiel ausgerichtet und als integraler Bestandteil des Sprechens und der Sprache anzusehen. “40 Dabei zeigt gestische Interaktion ein erhöhtes kommunikatives Bestreben - um sich im Gespräch mitzuteilen, den anderen zu verstehen, sich über eine „gemein-

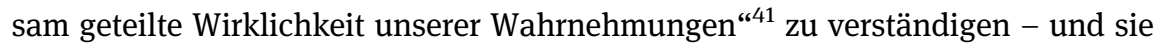
realisiert zugleich ein affektives Involviertsein, dessen Qualifizierung in den je spezifischen gestischen Bewegungsqualitäten liegt. ${ }^{42}$ Dass von den körperlichen Ausdrucksmöglichkeiten insbesondere die Geste in das Nachdenken über die Metapher mit einbezogen wird, ist instruierend, da mit der Geste eine dynamische, sinnlich-bildliche Darstellungsdimension und zugleich eine Expressivität und Affektivität in den Blick genommen wird, die auch für das audiovisuelle Bild zentral sind. Verkörperung wird hier nun in ein Konzept des Metaphorisierens integriert.

Da nun ,Multimodalität‘ - anders als gegenwärtig für Linguistik und Semiotik - für die Film- und Medienwissenschaft nicht zu den gängigen Termini gehört, sei hier eine Einordnung vorgenommen. ,Multimodalität‘ ist einer jener Sammelbegriffe, mit dem gegenwärtig auf vielseitige Weise gearbeitet wird, wobei die der Multimodalität zugrundeliegende Auffassung von ,Modalität‘ sich zumeist zwischen ,semiotischer Ressource' und ,adressierter Sinnesmodalität' bewegt. ${ }^{43}$ (Müllers Modalitätsbestimmung etwa orientiert sich hier an Zweiterem.) ${ }^{44}$ Die Multimodalitätsforschungen durchzieht, so resümiert es Henning Engelke, der explizite Anspruch, beim Verständnis von Kognition und (der Herstellung von) Bedeutung eine Fokussierung allein auf die Sprache und die damit verbundenen epistemischen Restriktionen zu überwinden. ${ }^{45}$ Dieser Anspruch geht häufig mit

39 Cornelia Müller: Mimesis und Gestik. In: Die Mimesis und ihre Künste. Hrsg. v. Gertrud Koch, Martin Vöhler und Christiane Voss. München: Wilhelm Fink 2010, S. 149-187, hier S. 150f. Vgl. auch Cornelia Müller: Redebegleitende Gesten. Kulturgeschichte, Theorie, Sprachvergleich. Berlin: Berlin-Verlag A. Spitz 1998.

40 Müller: Mimesis und Gestik, S. 150.

41 Kappelhoff: Kognition und Reflexion, S. 166, Herv. i.O.

42 Vgl. Müller/Kappelhoff: Cinematic Metaphor, S. 146.

43 Vgl. Bateman/Schmidt: Multimodal Film Analysis, S. 75.

44 Vgl. Cornelia Müller/Alan Cienki: Words, Gestures and Beyond. Forms of Multimodal Metaphor in the Use of Spoken Language. In: Multimodal Metaphor. Hrsg. v. Charles J. Forceville und Eduardo Urios-Aparisi. Berlin/New York: De Gruyter 2009, S. 297-328, hier S. 301.

45 Henning Engelke: Susanne K. Langer und Parker Tyler über Film als „,multimodales Medium“. In: Multimodale Bilder. Zur synkretistischen Struktur des Filmischen. Hrsg. v. Lars C. Grabbe, Patrick Rupert-Kruse und Norbert M. Schmitz. Darmstadt: Büchner 2013, S. 171-187, hier S. 171. 
einer weiteren, eher implizit bleibenden Zielsetzung einher. Vor dem Hintergrund wissenschaftlicher Ausdifferenzierung und Poststrukturalismus hat sich das Bedürfnis herausgebildet, eine ganzheitliche Perspektive wiederzugewinnen: „eine Perspektive, die Kognition und Bedeutung in der Gesamtheit ihrer perzeptiven, semantischen, semiotischen, diskursiven und kulturellen Dimensionen erfasst““. ${ }^{46}$ Damit setzen sich der Multimodalitätsdiskurs im weiteren Sinne und die Forschung zu verbal-gestischen Metaphern im Besonderen mit filmwissenschaftlich virulenten Themen auseinander - ist Film doch schon immer viel mehr und anderes als gesprochene (oder geschriebene) Sprache - und bieten Anknüpfungspunkte für eine film- und medienwissenschaftliche Auseinandersetzung mit Bedeutung, Verstehen und Denken im Kontext audiovisueller Bilder. ${ }^{47}$

Zum Auftakt der Erläuterung des Müller'schen Verständnisses von Metaphern im Sprachgebrauch sei mit Rudolf Arnheim nun jedoch zunächst einem Kunst-

46 Ebd. Für eine Übersicht zum Multimodalitätsdiskurs vgl. Janina Wildfeuer: Building the Gap between Here and There. Combining Multimodal Analysis from International Perspectives. In: Building Bridges for Multimodal Research. International Perspectives on Theories and Practices of Multimodal Analysis. Hrsg. v. Janina Wildfeuer. Bern/New York: Peter Lang 2015, S. 13-33, hier S. 13-24. Arbeiten, die sich unter dem Label der Multimodalität mit audiovisuellen Bildern beschäftigen, sind neben denen Forcevilles etwa Monografien und Sammelbände wie Jan Georg Schneider/Hartmut Stöckl (Hrsg.): Medientheorien und Multimodalität. Ein TV-Werbespot - sieben methodische Beschreibungsansätze. Köln: von Halem 2011; Bateman/Schmidt: Multimodal Film Analysis; Lars C. Grabbe/Patrick Rupert-Kruse/Norbert M. Schmitz (Hrsg.): Multimodale Bilder. Zur synkretistischen Struktur des Filmischen. Darmstadt: Büchner 2013; Wildfeuer: Film Discourse Interpretation. Für den Fernsehbereich ist etwa die Arbeit von Holly zu nennen, z. B. Werner Holly: Nachrichtenfilme als multimodale audiovisuelle Texte. In: Handbuch Sprache im multimodalen Kontext. Hrsg. v. Nina-Maria Klug und Hartmut Stöckl. Berlin/Boston: De Gruyter Mouton 2016, S. 392-409. Auch diese Publikationen bestätigen, dass Multimodalität vor allem für eine textlinguistisch und/oder semiotisch informierte Reflexion relevant ist.

47 Wie randständig das Diskursfeld der Multimodalität für die Filmwissenschaft ist, zeigt nicht zuletzt auch die Publikationsgeschichte des Bandes Medientheorien und Multimodalität: Bei der Konzeption der Tagung, die jener Publikation voranging, wurde die Filmwissenschaft nicht berücksichtigt (vgl. Jan Georg Schneider/Hartmut Stöckl: Medientheorien und Multimodalität. Zur Einführung. In: Medientheorien und Multimodalität. Ein TV-Werbespot - sieben methodische Beschreibungsansätze. Hrsg. v. Jan Georg Schneider und Hartmut Stöckl. Köln: von Halem 2011, S. 10-38, hier S. 17). Zugleich findet die filmwissenschaftliche Theoriebildung in der semiotisch und textlinguistisch geprägten Multimodalitätsforschung eher keinen Niederschlag. Dies zeigt sich etwa darin, dass von Filmen lediglich als bewegten Bildern gesprochen wird (vgl. etwa Hartmut Stöckl: Multimodalität - semiotische und textlinguistische Grundlagen. In: Handbuch Sprache im multimodalen Kontext. Hrsg. v. Nina-Maria Klug und Hartmut Stöckl. Berlin/Boston: De Gruyter Mouton 2016, S. 3-35; Holly: Nachrichtenfilme als multimodale audiovisuelle Texte). Gerade jedoch ein Verständnis filmischer Bilder als Bewegungsbilder ist für das Metaphorisieren zentral (vgl. Kapitel 8.3 der vorliegenden Arbeit). 
und Medientheoretiker das Wort gegeben, der nicht nur für die Filmwissenschaft wichtig ist, sondern der mit seinen Überlegungen zur Geste auch seinen Niederschlag in der Kognitiven Linguistik gefunden hat. Arnheim veröffentlicht 1969 mit seinem Buch Anschauliches Denken. Zur Einheit von Bild und Begriff eine Gegenrede zur Annahme, „daß die Sinneswahrnehmung und das Denken getrennte Gebiete seien““. ${ }^{48}$ In diesem Zusammenhang formuliert er seine wesentlich in der Gestalt- und Ausdruckstheorie ${ }^{49}$ gründenden Anmerkungen zu Eigenarten und Vermögen von Gesten, die eine erste Skizze davon geben, was Müllers Arbeiten zu Geste und multimodalen Metaphern zeigen: wie bei Metaphern im Sprachgebrauch mit der Sinnlichkeit der Geste Denken anschaulich wird.

Menschliche Gesten sind für Arnheim zunächst einmal Bilder - „Bilder, die [...] in die Luft geschrieben sind“. ${ }^{50}$ Doch es sind besondere Bilder, insofern man an ihnen beobachten kann, wie Begriffe Gestalt annehmen, wie Sinneswahrnehmung und Denken im alläglichen Gespräch konstitutiv zusammenhängen. Mit Arnheim lassen sich diesbezüglich verschiedene Vermögen und Eigenarten von Gesten festhalten:

- Wenn gestische Bewegungen wesentliche Bewegungsqualitäten und Dynamiken von Themen auswählen und hervorheben, geben sie mit ihrem körperlichen Ausdruck einen unmittelbaren Eindruck: „Gesten veranschaulichen Stoßen und Ziehen, Durchbruch und Hindernis, Klebrigkeit und Härte. “51

- Gestische Bewegungen sind nicht isoliert zu betrachten, sondern immer im situativen Kontext ihrer Begleitumstände: Denn es bleibe den „Begleitumständen“ überlassen, klarzustellen, worauf die Geste sich im Besonderen beziehe - „erstaunlich groß mag das Weihnachtspaket vom reichen Onkel oder auch der am vorigen Sonntag gefangene Fisch sein. “52

48 So Arnheim in seinem Vorwort (Rudolf Arnheim: Anschauliches Denken. Zur Einheit von Bild und Begriff [1969]. 7. Auflage. Köln: DuMont 1996, hier S. 9). Wie einige Jahre später auch Lakoff und Johnson, grenzt Arnheim sich also von der Annahme einer Dichotomie von Körper und Geist $\mathrm{ab}$ - er argumentiert jedoch nicht dekontextualisiert und mit Blick auf ein konzeptuelles System, sondern auf den Gebrauch bezogen.

49 Arnheims Ausdruckverständnis zeigt deutliche Parallelen zu dem von Helmuth Plessner: Mit ,Ausdruck“ werden alle „Modi organischen oder unorganischen Verhaltens“ gefasst, „die sich in der dynamischen Erscheinung von Wahrnehmungsobjekten oder -vorgängen offenbaren“. (Rudolf Arnheim: Kunst und Sehen. Eine Psychologie des schöpferischen Auges [1965]. 3., unveränd. Aufl. Berlin [u. a.]: De Gruyter 2000, hier S. 448, Herv. i. O.) Vgl. zu Plessner die Ausführungen zu „Ausdruckstheorie - Amodalität“ in Kapitel 8.3 der vorliegenden Arbeit.

50 Arnheim: Anschauliches Denken, S. 118.

51 Ebd., S. 117.

52 Ebd. 
- Durch die Begleitumstände können gestische Bilder Teil metaphorischer Vorstellungsszenarien sein: „Man kann mit Gebärden den Gang einer Auseinandersetzung darstellen, als wäre sie ein Boxkampf: das Abwägen der Angriffsmöglichkeiten, das Hin- und Herspringen, den geschickten Seitenangriff, den niederschmetternden Knockout. “53

- Die sich in Gesten abbildenden „Anschauungsqualitäten von Form und Bewegung“ sind konstitutiver Teil des Denkprozesses: „ja [...] diese Qualitäten [sind] selber das Medium [...], in dem sich das Denken abspielt. Natürlich handelt es sich hier nicht immer nur um visuelle Eigenschaften. Die Muskelempfindungen beim Stoßen, Ziehen, Vordringen und Widerstandleisten sind ein wesentlicher Bestandteil des Gebärdenspiels. “54

Um eine solche hier sehr verdichtet vorgebrachte Idee eines verkörperten Denkens, in das die Geste konstitutiv involviert ist, filmwissenschaftlich fruchtbar zu machen, bedarf es einer metapherntheoretischen Präzisierung, einer genaueren Taxonomie sowie einer Reflexion analytischer Verfahren. Dies ist mit Müllers Modell der Aktivierung von Metaphorizität möglich, dem ein Verständnis einer dynamischen Form des Konstiutierens von Bedeutung und einer „embodied form of understanding“ inhärent ist. ${ }^{55}$ In Anschluss an Karl Bühlers Organonmodell ${ }^{56}$ werden Sprache wie auch Geste dort mehrdimensional betrachtet: Es sind Artikulationsformen eines kommunikativen Ereignisses, die sich immer an jemanden richten (appellativer Charakter), die Handlungen, Objekte, ,Welt‘ zur Darstellung bringen (darstellender Charakter); und zugleich Haltungen involvieren, affektiv sind (expressiver Charakter). ${ }^{57}$ Dabei ist ein Unterschied zwischen Bühler und

53 Ebd.

54 Ebd., S. $117 \mathrm{f}$.

55 Cornelia Müller: Waking Metaphors. Embodied Cognition in Multimodal Discourse. In: Metaphor. From Embodied Cognition to Discourse. Hrsg. v. Beate Hampe. Cambridge: Cambridge University Press 2017, S. 297-316, hier S. 315 f.

56 Karl Bühler: Ausdruckstheorie. Das System an der Geschichte aufgezeigt [1933]. 2., unveränd. Aufl. Mit einem Geleitwort von Albert Wellek. Stuttgart: Fischer 1968; Karl Bühler: Sprachtheorie. Die Darstellungsfunktion der Sprache [1934]. Ungek. Neudr. der Ausg. Jena, Fischer, 1934. Stuttgart: UTB 1982.

57 Vgl. Cornelia Müller: Gestures as a Medium of Expression. The Linguistic Potential of Gestures. In: Body - Language - Communication. An International Handbook on Multimodality in Human Interaction, Vol. 38.1. Hrsg. v. Cornelia Müller, Alan Cienki, Ellen Fricke, Silva H. Ladewig, David McNeill und Sedinha Teßendorf. Berlin/New York: De Gruyter Mouton 2013, S. 202-217; Cornelia Müller: Gestural Modes of Representation as Techniques of Depiction. In: Body - Language Communication. An International Handbook on Multimodality in Human Interaction, Vol. 38.2. Hrsg. v. Cornelia Müller, Alan Cienki, Ellen Fricke, Silva H. Ladewig, David McNeill und Jana Bressem. Berlin/New York: De Gruyter Mouton 2014, S. 1687-1702. Sowohl der sich Artikulierende 
Müller hervorzuheben. Bei Bühlers Sprachmodell spielt der Symbolbegriff eine wesentliche Rolle. ${ }^{58}$ Anders bei Müller: Gerade indem sie die Geste in ihre Überlegungen zur Sprache miteinbezieht, und mit ihrem damit verknüpften Modell aktivierter Metaphorizität, wird der oft für selbstverständlich erachtete linguistische Grundsatz, dass Sprache etwas rein Arbiträres und Bildhaftigkeit keine relevante Spracheigenschaft wäre, substantiell kritisiert. ${ }^{59}$

Wie schon für Lakoffs und Johnsons Modell konzeptueller Metaphern sind auch für Müller alltägliche Redewendungen und metaphorische Ausdrücke ein zentraler Ausgangspunkt der Auseinandersetzung. Jedoch kommen sie nun in ihren spezifischen Diskurskontexten in den Blick, d. h. in verbal-gestischen Gesprächen mit zwei (oder mehr) Teilnehmern ${ }^{60}$ oder auch z. B. im Unterrichten von Tanz. ${ }^{61}$ Eben in Auseinandersetzung mit diesen Interaktionskontexten entwickelt Müller ihre Idee des Dynamischen der Metapher.

So beschreibt sie beispielsweise ein Gespräch zwischen zwei Frauen über deren erstmalige Erfahrungen, sich zu verlieben. Eine der beiden Frauen erzählt dabei von den Annäherungen zwischen ihr und ihrem späteren ersten Freund bei einem Schulausflug im Bus, und sie sagt: „auf der Hinfahrt [...] hat es angefangen, dass es zwischen uns irgendwie gefunkt hat“. Während dieser sprachlichen Äußerung geschieht gestisch Folgendes:

Just as she is uttering the word ,gefunkt ${ }^{\star}[. .$.$] , she performs the brief, accentuated outward$ movement of a ring gesture. In this gesture, the index finger and the thumb touch each other at the fingertips as if they were picking up a tiny little object and form the shape of a ring. Her right hand moves into the ring configuration as she begins to talk about what happened between them (,zwischen uns') on this bus ride. Initially, the ring gesture is performed in a somewhat unusual position, namely, with the fingertips directed toward the speaker. As soon as she utters the word ,gefunkt', she quickly turns it outward, holds it for a short moment (now it is in a standard orientation with the fingers directed away from speaker),

als auch der, an den sich die sprachlichen und gestischen Artikulationen richten, werden als aktive Teilnehmer eines kommunikativen Ereignisses betrachtet, die die Artikulationsweisen wie auch deren Verständnis formen. Vgl. Müller: Gestures as a Medium of Expression, S. 204.

58 Vgl. Bühler: Sprachtheorie, S. 28.

59 Vgl. Müller: Metaphors Dead and Alive, Sleeping and Waking, S. xv.

60 Vgl. bspw. ebd; Cornelia Müller/Susanne Tag: The Dynamics of Metaphor. Foregrounding and Activation of Metaphoricity in Conversational Interaction. In: Cognitive Semiotics 10.6 (2010), S. 85-120.

61 Cornelia Müller/Silva H. Ladewig: Metaphors for Sensorimotor Experiences. Gestures as Embodied and Dynamic Conceptualizations of Balance in Dance Lessons. In: Language and the Creative Mind. Hrsg. v. Mike Borkent, Barbara Dancygier und Jennifer Hinnell. Stanford: CSLI 2014, S. 295-324; Müller/Kappelhoff: Cinematic Metaphor. Auch mit Text-Bild-Metaphern hat Müller sich beschäftigt (vgl. Müller: Metaphors Dead and Alive, Sleeping and Waking). 
and as the metaphorical lexeme ends, she releases the ring configuration, and moves her hand downward into a resting position. ${ }^{62}$

Was sich hier als kommunikative Handlung im Zusammenspiel von Sprache und Geste vollzieht, ist Müller zufolge zweierlei. Mit der gestischen Ring-Konfiguration wird das kleine Objekt dargestellt, von dem auch sprachlich die Rede ist: der elektrische Funke. Und mit der spezifischen Art und Weise, mit dem die Ringgeste in ihrer räumlichen Orientierung transformiert wird - einer schnellen Bewegung aus dem Handgelenk -, wird das Bewegungsmuster, die Bewegungsdynamik dieses Funkens vollzogen, zum Ausdruck gebracht. ${ }^{63}$

Müller zufolge zeigt sich darin, dass der Bildgehalt von konventionalisierten Sprachmetaphern ,erwachen' kann. Ihre Gesprächsbetrachtung macht deutlich, dass die klassische These des Totseins konventionalisierter Metaphern ${ }^{64}$ sich aus Sicht des Sprachgebrauchs als nicht haltbar erweist. Denn die Situation ,berühren sich zwei elektrisch geladene Entitäten, entlädt sich elektrische Spannung in einem Funken', die mit der konventionalisierten metaphorischen Redewendung „es hat gefunkt" für das Moment des Sich-Verliebens sprachlich adressiert wird, wäre als Erfahrungsbereich ansonsten nicht (mehr) zugänglich. Doch dass für die Gesprächsteilnehmer in dem oben beschriebenen Gespräch Zugang zu diesem Erfahrungsbereich besteht - eben dies zeigt sich im spontanen Gestikulieren, in der Artikulation dieser multimodalen Metapher, einer Metapher, in deren Artikulation mehrere expressive Modalitäten involviert sind. ${ }^{65}$ Ein solches Zusammenspiel von Sprechen und Gestikulieren wird daher als kognitiver Prozess, der empirisch beobachtbar ist, aufgefasst - ein Prozess der Aktivierung von Metaphorizität, der mit einer intersubjektiven Aufmerksamkeitslenkung auf Metaphorizität einhergeht. ${ }^{66}$

Wenn in diesem Zuge vom Aufwachen schlafender Metaphern die Rede ist, dann sind damit Metaphern gemeint, deren Metaphorizität erst einmal grund-

62 Müller: Metaphors Dead and Alive, Sleeping and Waking, S. 34.

63 Siehe ebd.

64 Vgl. hierzu auch die Erläuterungen in Kapitel 2 der vorliegenden Arbeit.

65 Ein solches Verständnis einer multimodalen Metapher ist entsprechend anders gelagert als die von Forceville vorgeschlagene Definition (,multimodal metaphors are metaphors whose target and source are each represented exclusively or predominantly in different modes“, Forceville: Non-Verbal and Multimodal Metaphor in a Cognitivist Framework, S. 24).

66 Vgl. insbesondere Müller: Metaphors Dead and Alive, Sleeping and Waking; Müller/Tag: The Dynamics of Metaphor; Cornelia Müller: Reaction Paper. Are ,Deliberate‘ Metaphors Really Deliberate? A Question of Human Consciousness and Action. (Online announced as: Are ,Deliberate' Metaphors Really Special? Deliberateness in the Light of Metaphor Activation). In: Metaphor and the Social World 1.1 (2011), S. 61-66; Müller: Waking Metaphors. 
sätzlich für durchschnittliche Sprecher/Zuhörer in einer Gesprächssituation transparent und daher verfügbar ist. ${ }^{67}$ Entsprechend wird die für eine Gebrauchsperspektive dysfunktionale Kategorisierung ,tote Metapher vs. lebendige Metapher' verabschiedet und die Frage, ob man es mit einer konventionalisierten oder mit einer neuen Metapher zu tun hat, zweitrangig, beziehen sich solche Kategorisierungen doch weniger auf die Ebene des Sprachgebrauchs in konkreten Interaktionen als vielmehr auf die des kollektiven Sprachsystems. Die Aktivierung von Metaphorizität hingegen ist - wie auch das Verständnis metaphorischer Kreativität, das Camerons Modell inhärent ist - auf der Ebene konkreter Artikulationen verortet und somit immer kontextsensitiv.

Die Beobachtung, dass in der Alltagskommunikation Erfahrungsbereiche, wie sie in sprachlich artikulierten Metaphern zum Ausdruck kommen, gestisch aktiviert werden, führt Müller zu folgender These: Sprachlich ausgedrückte Metaphern seien nicht entkörperlichte, abstrakte (man könnte ergänzen: gänzlich arbiträre) Repräsentationen von Erfahrungsbereichen oder abstrakten lexikalischen Feldern, so Müller. Vielmehr seien sie körperlich ,bewohnt‘: [...] „,verbally expressed metaphors are ,inhabitated' bodily; [...], they function as a ,door opening“, an invitation for the speaker“ - und für das zuhörende Gegenüber - „to move into an imaginary world [...]."68 (Auch wenn dies spekulativ bleiben muss - da unklar bleibt, ob Arnheims Beispiele konstruiert sind beziehungsweise, falls nicht, wie genau sich die herangezogenen Situationen dargestellt haben mögen -: Im Falle von Arnheims Boxkampf-Beispiel könnte etwa die Schilderung eines ,schlagkräftigen Arguments' eine solche Einladung in eine Vorstellungswelt gewesen sein.) $\mathrm{Zu}$ dieser körperlichen Sprachauffassung Müllers kommt hinzu, dass die Anschauungsformen von Gesten - wie unten ausführlich dargelegt werden wird ${ }^{69}$ - immer auch verkörperte Erfahrungen, subjektive „felt sensations“ der gestisch artikulierten metaphorischen Erfahrungsbereiche (mit Blick auf die obigen Beispiele etwa der des Funkens oder der des Boxens) zum Ausdruck bringen, die intersubjektiv im wahrnehmenden Gegenüber körperliche Resonanz finden: ein jeweils in der spezifischen Ausführungsart, in der Bewe-

67 „Depending on the flow of interaction and the respective contexts of use, the same verbal metaphor can be sleeping at one point in discourse and waking at another. This potential for metaphor activation by discourse contexts only disappears when metaphors stop to be transparent to their users. Only if a historical metaphor cannot be activated by an average speaker because the experiential source domain that inspired the metaphoric mapping is no longer part of a shared language and culture, can this metaphor be appropriately characterized as ,dead“.” (Müller: Waking Metaphors, S. 299). Vgl. für eine grafische Darstellung auch Abb. 51 (im Anhang).

68 Müller: Metaphors Dead and Alive, Sleeping and Waking, S. 94.

$69 \mathrm{Vgl}$. Kapitel 8.3 der vorliegenden Arbeit. 
gungsqualität und Dynamik der Gesten gründendes verkörpertes, interaffektives Erleben. ${ }^{70}$ „An der gestischen Interaktion in konkreten Konversationen wird also ablesbar“, so resümiert Kappelhoff die Forschung Müllers,

dass Prozesse der Metaphorisierung grundlegende affektive Vektoren in das Gespräch einbringen, die den aktuellen Kommunikationsverlauf einbetten in eine gemeinsam geteilte imaginäre Wahrnehmungsszene. Diese kann auch dann im gestischen Ausdruck als affektiv-sinnlicher common ground greifbar werden, wenn sie nicht verbal expliziert und keinem Gesprächspartner bewusst ist. ${ }^{71}$

Hierin liegt - um einen Blick vorauszuwerfen - ein für die späteren Ausführungen zum filmischen Metaphorisieren wesentlicher Punkt, denn auch

[f]ilmische Bewegungsbilder fungieren [...] als ein ad hoc sich formierender common ground geteilter Erfahrung, ein allen Zuschauern gleichförmig zugängliches - weil automatisch projiziertes - Wahrnehmungserleben, auf das sich die metaphorische Konstruktion stützen kann. $^{72}$

Eng damit zusammen hängt die Müller’sche Idee des „metaphorical scenario“. ${ }^{73}$ Diese fasst Sarah Greifenstein wie folgt zusammen:

Das ,metaphorical scenario' zeichnet sich dadurch aus, dass es als zeitliche Reihe von metaphorischen Äußerungen aus einem ähnlichen Bildbereich beschreibbar ist. Nach und nach entfaltet sich ein Szenario, wird konkreter, plastischer, vitaler. Der Gebrauch von Metaphern ist darauf ausgerichtet, das Vorstellungsbild von einer Sache in einer ganz bestimmten Hinsicht auszuschmücken, zu charakterisieren oder begreifbar zu machen. Müller betont vor allem den Prozess, wie ein solches Szenario gestisch und sprachlich hergestellt wird, wie sich langsam eine imaginäre Welt öffnet. Nicht das Resultat einer Metapher ist entscheidend, sondern die zeitliche Entfaltung, Anordnung und Aktivierung, wodurch eine Metapher über die Zeitstrecke eines Gesprächs immer wieder durch Rück- und Vorgriffe hervorgehobene Momente miteinander zu verbinden vermag. ${ }^{74}$

70 Vgl. Müller: Waking Metaphors, S. 299 sowie Kappelhoff/Müller: Embodied Meaning Construction. Vgl. ausführlich zur Geste in der Face-to-Face-Kommunikation als Ausdrucksbewegung Dorothea Horst/Franziska Boll/Christina Schmitt/Cornelia Müller: Gesture as Interactive Expressive Movement. Inter-Affectivity in Face-to-Face Communication. In: Body - Language - Communication. An International Handbook on Multimodality in Human Interaction, Vol. 2. Hrsg. v. Cornelia Müller, Alan Cienki, Ellen Fricke, Silva H. Ladewig, David McNeill und Jana Bressem. Berlin/New York: De Gruyter Mouton 2014, S. 2112-2125; Müller/Kappelhoff: Cinematic Metaphor, S. 146-154.

71 Kappelhoff: Kognition und Reflexion, S. 168.

72 Ebd., S. 139.

73 Müller: Metaphors Dead and Alive, Sleeping and Waking, S. 89.

74 Sarah Greifenstein: Tempi der Bewegung - Modi des Gefühls. Zur zeitlichen Organisation audiovisueller Ausdrucksbewegungen in Screwball Comedies (Manuskriptfassung), Freie Univer- 
Für audiovisuelle Bilder können Redewendungen ebenfalls eine Rolle spielen (wie schon die in die vorliegende Arbeit einführende Analyse des Mercedes-Spots und dessen Zeile „upside down you're turning me“ gezeigt hat). Dennoch ist es gerade aufgrund des filmwissenschaftlichen Kontexts, in dem die Ausführungen zu Müller hier erfolgen, wichtig, festzuhalten, dass das Entstehen und Entfalten metaphorischer Szenarien nicht an das Erwachen von Bildgehalten konventionalisierter sprachlicher Metaphern gebunden ist, sondern sich auch unabhängig von solchen Sprachäußerungen ereignet. ${ }^{75}$

Das Müller'sche Modell situiert sich durch seine Auseinandersetzung mit der Geste wie auch seine Sprachauffassung im Forschungsfeld zu Metapher und verkörperter Kognition, dem etwa auch die psychologischen Forschungen von Raymond W. Gibbs Jr. zuzuordnen sind. ${ }^{76}$ Darüber hinaus harmoniert das Modell mit dem Camerons, ähneln sich doch beide darin, wie auch Müller unterstreicht, „how metaphor works and emerges from discourses rather than being instantiated“. ${ }^{77}$ So wird in „der Dynamik gestischer Interaktion wie in der Dynamik des Diskurses“, wie Kappelhoff hervorhebt,

„deutlich, dass Metaphern im selben Maße Koproduktionen der Akteure einer aktuell gegebenen Interaktion sind, wie sie als strukturierende Faktoren solcher Interaktionen fungieren. [...] Metaphern lassen im Zwischenraum des wechselseitigen Unverständnisses einen gemeinsam geteilten Grund entstehen [...]." ${ }^{\text {78 }}$

Analytisch zeigt sich die gegenseitige Anschlussfähigkeit, wenn etwa ,Elaborierung' und ,semantische Opposition' nicht nur für Cameron zentrale Phänomene sind. Denn was bei jener als häufig vorkommende Entwicklungsformen metaphorischer Vehikel beschrieben und als Aktivität des Metaphorisierens begriffen wird, fasst Müller als Indikatoren aktivierter Metaphorizität: „[...] waking meta-

sität Berlin (Dissertation) 2015, hier S. 108; für Analysen von Face-to-Face-Interaktionen hierzu vgl. vor allem auch Müller/Kapppelhoff: Cinematic Metaphor.

75 Vgl. z. B. die,Holy Aura'-Analyse in Müller/Tag: The Dynamics of Metaphor, S. 103-111.

76 Vgl. hierzu insbesondere auch die Publikationen von Gibbs: Raymond W. Gibbs, Jr.: Why Idioms Are Not Dead Metaphors. In: Idioms: Processing, Structure, and Interpretation. Hrsg. v. Cristina Cacciari und Patrizia Tabossi. Hillsdale: Erlbaum 1993, S. 57-78; Gibbs: Embodiment and Cognitive Science; Raymond W. Gibbs, Jr.: Embodied Metaphor. In: The Bloomsbury Companion to Cognitive Linguistics. Hrsg. v. Jeanette Littlemore und John R. Taylor. London: Bloomsbury 2014, S. 167-184.

77 Cornelia Müller/Christina Schmitt: Audio-Visual Metaphors of the Financial Crisis. Meaning Making and the Flow of Experience. In: Revista Brasileira de Linguística Aplicada/Brazilian Journal of Applied Linguistics 15.2 (2015), S. 311-341, doi: http://dx.doi.org/10.1590/1984-639820156315, hier S. 313.

78 Kappelhoff: Kognition und Reflexion, S. 168f. 
phors are surrounded by metaphoricity indicators, such as verbal elaboration, specification, semantic opposition, syntactic integration, or coexpressions of metaphor in a cooccurring modality such as gesture [...].“79

Die Indikatoren aktivierter Metaphorizität sind also im Ausdruck gegeben, dort zeigen sich kognitive Prozesse. Dabei beansprucht Müllers Modell nicht, auf einer psychologischen Ebene Aussagen über Kognition zu machen. Vielmehr wird damit eine Idee anschaulichen Denkens im Sinne Arnheims weiterentwickelt. Gerade indem die Geste in die theoretische Argumentation einbezogen wird, geht Müller mit ihrer Auffassung von Metaphern als „embodied cognition in discour$\mathrm{se}^{\text {“80 }}$ weiter als Cameron:

[...] in contrast to Cameron's focus on linguistic metaphor, the dynamic view on metaphor inherently addresses the multimodal nature of metaphoric meaning making in communication. It doing so, it shifts scope, as it assumes that multimodal metaphors [...] involve an actual experiencing of metaphoricity and not only a potential emergence of discourse metaphor, as is assumed by Cameron's approach. ${ }^{81}$

In einem solchen Verständnis erscheinen verbal-gestische Metaphern als Prozesse anschaulichen Denkens - und dies in doppelter und miteinander verschränkter Weise: Das Anschauliche und Sinnliche liegt im Metaphorischen wie auch darin, dass die Ausdrucksebene dabei eine so zentrale Rolle spielt. Methodisch ermöglicht dies einen deskriptiven Zugang zu verkörpertem Denken. Denn über das Zusammenspiel von Sprechen und Gestikulieren lässt sich beobachten, wie Metaphern kontextgebunden emergieren.

Auch bei Müller ist damit von ,Metapher“ im Sinne eines Metaphorisierens die Rede, denn ,Metapher‘ meint, wie bereits erwähnt, immer einen Prozess und also kein Produkt. Metaphorisieren bei Müller meint den Prozess des Verbindens von zwei Bereichen. Das heißt, dass nicht allein die Involvierung zweier Bereiche für eine Metapher relevant ist. Entscheidend ist, sich zu vergegenwärtigen, dass man es mit einer zwischen diesen Bereichen „vermittelnden Kraft“ bzw. mit einem die Bereiche „verbindenden Prozess“ zu tun hat. ${ }^{82}$ Eben dies ist das „seeing as“, das „seeing one thing in terms of another“: ${ }^{83}$ Metaphorisieren ist der sich in der Zeit entfaltende und beobachtbare Prozess des „seeing and feeling one experiential

79 Müller: Metaphors Dead and Alive, Sleeping and Waking, S. 198.

80 Müller: Waking Metaphors, S. 316, Herv. i. O.

81 Müller/Schmitt: Audio-Visual Metaphors of the Financial Crisis, S. 313. Vgl. auch Müller: Metaphors Dead and Alive, Sleeping and Waking, S. 198.

82 Vgl. Müller: Metaphors Dead and Alive, Sleeping and Waking, S. 26.

83 Siehe ebd. 
domain in terms of another“. ${ }^{84}$ Wenn von der Aktivierung, der Etablierung von Metaphorizität die Rede ist, dann ist damit notwendiger Weise auch immer von diesem Prozess die Rede. ${ }^{85}$

In analytischer Perspektive operiert die von Müller verfolgte Idee der Dynamik solcher Prozesse sowohl auf einer horizontalen wie auch auf einer vertikalen Ebene, da sich die jeweilige Charakteristik der Metaphorizitätsaktivierung in der zeitlichen Entwicklung diskursiver Ereignisse und dem jeweiligen kommunikativen Aufwand zeigt, durch den metaphorische Erfahrungsbereiche wahrnehmbar werden. ${ }^{86}$ Infolgedessen ist Metaphorizität auch ein graduelles Phänomen - denn je mehr Metaphorizitätsindikatoren beobachtbar sind, desto stärker ist ein metaphorisches Erfahrungsmoment gegeben. Im Rahmen eines multimodalen Diskursereignisses manifestiert sich das Aktivieren von Metaphorizität daher „as a dynamic and temporally orchestrated profiling of multimodal utterances“. ${ }^{87}$

Vor diesem Hintergrund lässt sich postulieren, dass Arnheims Beschreibung des Boxkampf-Beispiels auf ein Diskursereignis mit einer relativ starken Aktivierung von Metaphorizität durch verbal-gestische Elaborierung hindeutet. Eine Schlussfolgerung, die Arnheim aus dem Boxkampf-Beispiel zieht, erscheint allerdings zu weitreichend bzw. allgemein, da letztlich doch zu wenig aus Diskursperspektive gedacht. So heißt es bei Arnheim, eine „spontane Verwendung von Metaphern“ zeige, „daß die Menschen sich überall der strukturellen Ähnlichkeit bewußt sind, die physische und nichtphysische Dinge verbinden“. ${ }^{88}$ Müllers Modell aktivierter Metaphorizität impliziert hingegen weniger die These einer grundsätzlich bewussten Metaphorizität. ${ }^{89}$ Vielmehr macht dieses Modell stark, dass die jeweils spezifischen Artikulationsweisen Aufschluss darüber geben, ob und in welcher hervorgehobenen Weise „metaphoricity is an active aspect of meaning at a particular moment in a discourse event“..$^{90}$

84 Müller: Waking Metaphors, S. 300.

85 Vgl. Müller: Metaphors Dead and Alive, Sleeping and Waking, S. 30.

86 Vgl. Müller/Schmitt: Audio-Visual Metaphors of the Financial Crisis, S. 314.

87 Müller/Tag: The Dynamics of Metaphor, S. 93; vgl. auch Müller: Metaphors Dead and Alive, Sleeping and Waking, S. 198.

88 Arnheim: Anschauliches Denken, S. 117.

89 Vgl. zur Debatte zu Metapher und Bewusstheit Müller: Waking Metaphors.

90 Ebd., S. 299. 


\subsection{Resümee: ,Metapher` in der Alltagskommunikation meint das dynamische Phänomen des Metaphorisierens}

Die Darlegungen Camerons und Müllers reflektierend, seien zum Abschluss dieses Kapitels zu sprach- und gestenwissenschaftlichen Bezugspunkten eines vom Gebrauch ausgehenden Metaphernverständnisses nun noch einmal zentrale Aspekte thesenhaft gebündelt:

- Die Idee eines Systems spielt für das Metaphorisieren nicht als genealogisches, vom Gebrauch instanziiertes Moment eine Rolle. Wohl aber werden Diskursereignisse als dynamische Systeme diskutiert. Als in der Zeit sich ereignende, entstehende, entfaltende Ganzheiten. Als Interaktions- und Austauschprozesse, in denen Dynamiken und Kräfte zusammenwirken und fluide Relationen, Formen, Aufmerksamkeiten, Wahrnehmungen ausbilden.

- Von einem Ereignis zu reden, meint hier etwas, das immer schon an seine Materialität, seinen Ausdruck, seine Zeit gebunden ist, nicht unabhängig davon existiert. Vor diesem Hintergrund meint ,Metapher‘ situativ verortete, diskursimmanente Prozesse, die zugleich die Situation, den Diskurs, das Ereignis auch erschaffen.

- Metaphern emergieren, entfalten sich im Zeitverlauf, sind dynamisch, bilden Muster aus - in einem solchen Sinne ist die Metapher diskursgestaltend. ,Metapher‘ ist zudem nicht ohne die Kraft, den Akt des Verbindens zu denken, nicht ohne die Aktivität des Eins-durchs-Andere-Sehens, nicht ohne einen Wahrnehmungsvollzug. Vom Gebrauch aus gesehen, liegt eben hierin das Schöpferische, das Kreative der Metapher - im Sinne eines Metaphorisierens.

- Ein Metaphorisieren ist an den Artikulationen selbst zu beschreiben - und lässt sich, bezogen auf das jeweilige Diskursereignis, auf einer Makro- wie Mikroebene untersuchen. Untersucht werden nicht Produkte, sondern Prozesse - des Kommunizierens, des wechselseitigen Verstehens und geteilter Wahrnehmungen, des verkörperten Denkens, des Herstellens von Bedeutung.

- Das Zusammenspiel von Geste und Sprache artikuliert und entfaltet metaphorische Wahrnehmungs- und Vorstellungsszenarien. Hierbei spielt nicht nur das Vermögen der Geste eine Rolle, auf dynamische Weise bildhaft darzustellen, zentral ist ihr immer schon verkörpertes, expressives, intersubjektives Wesen. Gesten stiften im Metaphorisieren einen sinnlich-affektiven common ground.

- In shiftings und multimodalen Artikulationen zeigt sich so ein Metaphorisieren: ein Wahrnehmungsvollzug, ein Sehen, Fühlen und Verstehen eines 
Erfahrungsbereichs durch einen anderen, ein anschauliches Denken in dynamischen Bildern.

Diese Aspekte sind wegweisend für ein in der Gebrauchsdimension verankertes Verständnis des Phänomens ,Metapher‘ im Kontext audiovisueller Bilder. Doch inwiefern kann ein Verständnis filmischen Metaphorisierens hieran theoretisch wie methodisch anknüpfen? Um dies zu eruieren, ist es erforderlich, sich genauer über die Beschaffenheit filmischer Kommunikation zu verständigen. Damit ist jetzt das in expliziter Weise metapherntheoretische Feld $\mathrm{zu}$ verlassen und ein medien- und ausdruckstheoretisches Feld zu betreten: ein Feld, das die These durchzieht, dass es bei der Rezeption audiovisueller Bilder immer schon um Ausdruck, Wahrnehmung und Verkörperung geht - und eben darin das Movens für Bedeutung und Verstehen liegt. Im Zentrum wird die gestische Dimension audiovisueller Bilder stehen, die sich schließlich mit einer Idee des Metaphorisierens verschränkt. Und so, wie für Cameron und Müller die Idee der Emergenz anstelle der Instanziierung zentral ist, distanziere auch ich mich im Folgenden vom Zwei-Welten-Modell. 